\title{
A Novel Technique for Analysing Performance using Conceded Cluster Heads in VANETs
}

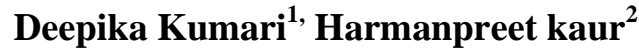 \\ M. Tech Student, Department of Computer Science \& Engineering, Sri Sai College of Engineering and Technology, \\ Amritsar, Punjab, India ${ }^{1}$ \\ Assistant Professor, Department of Computer Science \& Engineering, Sri Sai College of Engineering and Technology, \\ Amritsar, Punjab, India ${ }^{2}$
}

\begin{abstract}
In recent era, vehicles have an eminent role in the modern society. Umpteen researchers using VANET domain for vehicles making possible dynamic path scheduling in the current decade. VANET comprises V2V include only transmission between vehicle to another vehicle and the data is transmitted with the help of On Board Unit (OBU). An On-board Unit is an equipment that exist in vehicle and aids in distribution of information with Road Side Units or with additional On Board Units. In addition to V2I, the communication takes place between vehicles and Road Side Units (RSU) and RSU act as infrastructures. VANET protocol delivers data packets to vehicles in a short span of time. A vehicle in VANET is taken into account to be an intelligent mobile node capable of communicating with its neighbours and alternative vehicles within the network. The main purpose to utilize this active area of improving safe driving, traffic optimization, coupling and some other services. It plays an important role in intelligent transportation system. To perform better in large scale environment the clustering is proposed. In clustering the mobile node is grouped within range and one mobile node is elected as cluster head which is responsible for transmission in cluster. The proposed Technique work on dynamic cluster development in which the node within area are combined together and group of node is designed called cluster. In cluster formation process the energy, speed and range of each node is calculated using weighted control matrices is called Composite Value (CV). On the basis of CV cluster head selection process is done and cluster is formed. In this technique Cluster Head $(\mathrm{CH})$ and Conceded Cluster Head $(\mathrm{CCH})$ are selected with the highest value of CV. It rescue the overall network channel and protect the routing path from link breakage. This technique outperforms the previous technique in the sense of packet delivery factor, delay and throughput.
\end{abstract}

Keywords: Composite value, Cluster, Cluster head, VANET

\section{INTRODUCTION}

In Vehicular Ad-hoc network considered as the intelligent mobile node by which the communication takes place between nodes and their neighbouring nodes. VANET contemplate the independent network by which the several routing protocols are operated. VANET network has ability to decide behaviour of routing system, mobility range of vehicle and arise efficient communication between the nodes. Due to decentralized nature of VANET, it uses low bandwidth, wireless sensors and also checks quality of services up to the date. VANET is differentiated into two categories: V2V and V2I. In V2V, vehicles act as nodes and data is transmitted between node to node. It may be more than one node which communicates with one another. The communication occurs between node to node through On Board Unit (OBU). OBU is an electronics device or sensor from which it senses the current node is passed through the mobile node. In case of V2I, there is exchange of information across the vehicles and infrastructure. The packets are sent from source to destination via Road Side Unit (RSU). RSU react as infrastructure, which is a fixed communicating antenna present at the road for wireless connection. VANET is self establishing group or self organising channel and also act as client- server network when it on demand.

\section{PREVIOUS WORK}

The previous paper demonstrates Cluster Based Routing Protocol. It works on the CBR algorithm. In this method, CBR algorithm geographically differentiates the regional zone into $\mathrm{W}^{2}$ grids. The $\mathrm{W}$ depicts the side length of the grid. The vehicles which are present in the grid sector are proposed as clusters. The data is exchanged with the help of cluster heads from source to destination. In this, it also selects the optimal cluster head through the angle enclosed by two rays. It elects the minimal path of angle which is covered by node. All in all, it was dependent on area enclosed of side grids. 
Problem Description: It geographically covered the area so we can say that it is only location based.

- $\quad$ It is not fully documented and has not fairly sensory experience.

- Link breakage does not maintained. It does not observe more results in single time slot.

- $\quad$ It covers long path but sometimes we have not too much time for cover such distance.

- Due to hold up of bulk area, sometimes packets discarded or intruders may present in source to destination.

- High energy consumption.

- If cluster routing table has no legal information of neighbouring nodes, then communication problem occurs.

- $\quad$ Most of the time, if message given to inaccurate client it may cause swear complications.

- $\quad$ Large bandwidth used.

\section{PROPOSED METHODOLOGY}

Our proposed Technique work on dynamic cluster development in which the node within area are combined together and group of node is called cluster. In cluster formation process the energy, speed and range of each node calculated in the form of weighted control matrices and it is called composite value. On the basis of Composite Value cluster head selction process is done and cluster is formed. In our proposed technique, Cluster Head and Conceded Cluster Head are selected with the highest value of Composite Value. The two nodes with the highest value of Composite Value are considered as Cluster Head and Conceded Cluster Head that are Composite Value 1 and Composite Value 2 respectively.

Step 1: Cluster formation: - Initiate the network and dividing the whole network in some disjoint group called cluster.

Step 2: For every node, weighted control matrices will be calculated. Weighted control matrices denoted as Composite Value here. The Composite Value can be calculated on the basis of maximum energy and speed and range. Among the all nodes in cluster, find two nodes with higher composite value, and name these two nodes as Cluster Head, Conceded Cluster Head.

Step3: Broadcast message nodes with highest Composite Value as Cluster Head and Conceded Cluster Head.

Step4: The primitive node will find the energy of non-cluster node, Cluster Head and Conceded Cluster Head. If energy of Cluster head goes down then primitive node will replace Conceded Cluster Head with Cluster Head and Conceded Cluster Head with cluster member node having highest energy find by primitive selection technique.

Step5: If in case cluster head leaves and departed, the conceded cluster head node will elected as cluster head and Primitive node selection algorithm will replace the node.

\section{PROPOSED ALGORITHM}

The proposed algorithm of dynamic technique for efficient dissemination to tackle the unstable topology of VANETs NN: Normal Node.

PN: primitive Node.

CV: Composite Value (Range, speed, energy).

$\mathrm{CH}$ : Cluster Head.

$\mathrm{CCH}$ : Conceded Cluster Head.

$\mathrm{SN}$ : next selected CCH Node.

\section{Cluster Formation}

In proposed algorithm the $\mathrm{CCH}$ will increase the life time of cluster for efficient routing.

Step 1: Begin

Step 2: Initiate the network with NN

\{

Step 3: Normal Node (NN) participates for the cluster head elections and finds the two Highest CV nodes.

Step 4: Node with highest CV will be elected as cluster head and second as $\mathrm{CCH}$.

Broadcast $\mathrm{CH}$ and $\mathrm{CCH}$.

Step 5: Primitive selection done for finding node with highest energy will be saved as PN.

Cluster is formed; Transmission in network

II Cluster Maintenance

Step 1: Transmission Cluster Formation

If ( $\mathrm{CH}$ leaves the network)

\{

The $\mathrm{CCH}$ node will be replaced with $\mathrm{CH}$ and become Cluster head. \} 
Step 2: Update $\mathrm{CCH}$ with Selected Node for new $\mathrm{CCH}$

Broadcast $\mathrm{CH} \& \& \mathrm{NACH}$ in network update routing table;

Step 3: Transmission in network;

\}

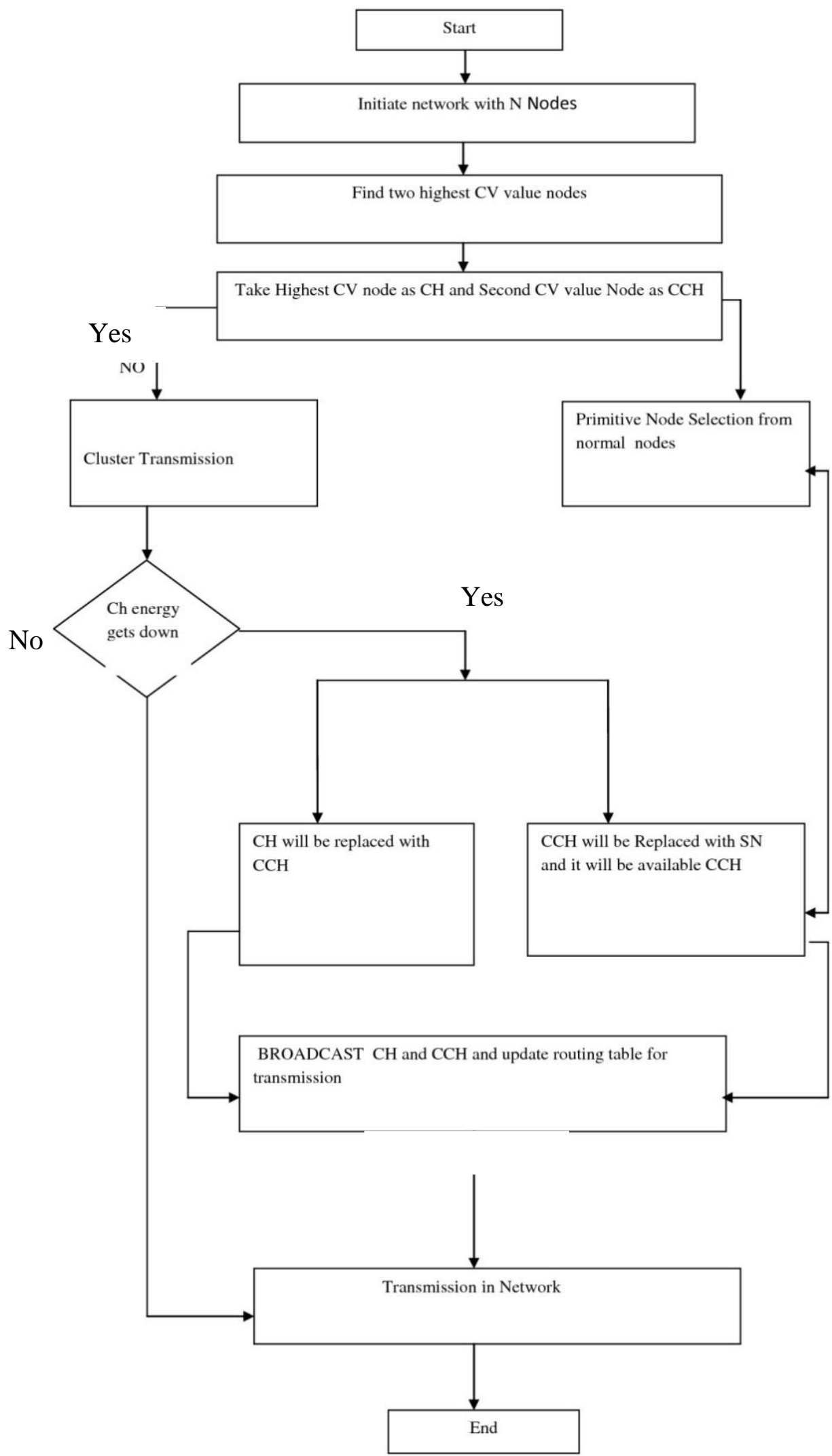




\section{RESULT ANALYSIS}

1. Packet delivery Factor: The PDR is the ratio of packet received by node from source to destination.The formula for calculation $\mathrm{PDR}=\mathrm{P} 1 \div \mathrm{P} 2$, where $\mathrm{P} 1$ and $\mathrm{P} 2$ are sum of packets generated by source and destination. The proposed technique result shown in Fig 1 with the increase of time packet delivery factor is better as compared to previous CBR technique.

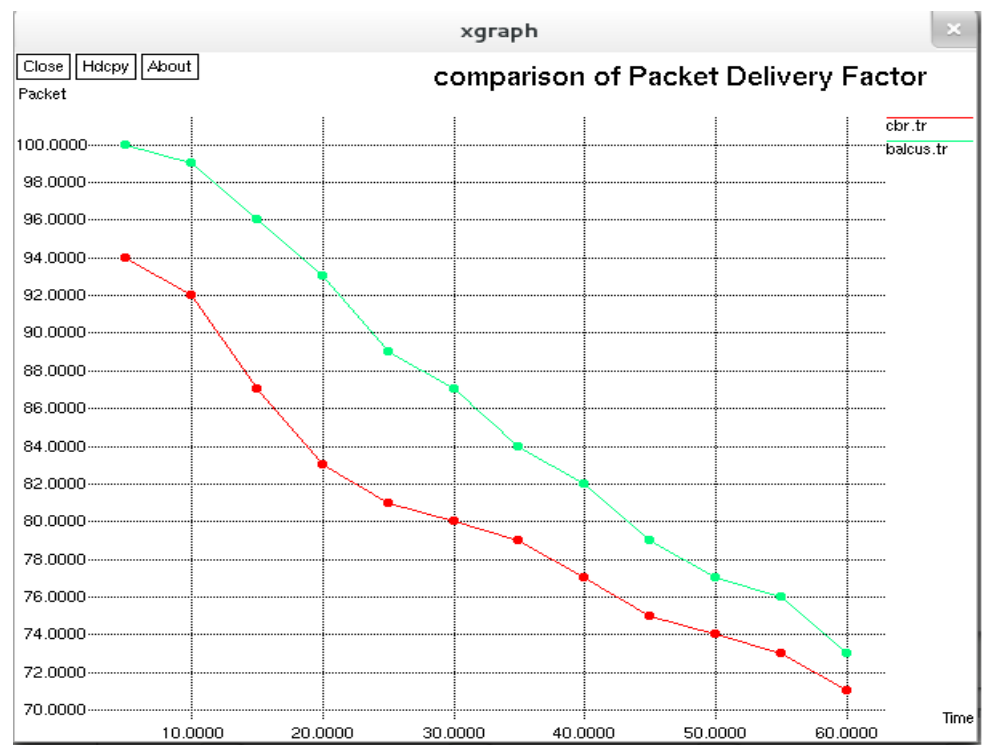

Fig 1 comparison of Packet Delivery ratio

2. Energy: The energy consumption is equal to the sum of energy units used by each transmission to retransmit the data to its members. This minimization of retransmission will decrease the total energy consumption.

\section{Energy consumed by node=initial energy of node - final energy of node}

The energy consumption of proposed technique after cluster formation and transmission shows that proposed energy is better. The result shown in fig 2 shows proposed technique energy consumption is lower as compared to previous CBR technique.

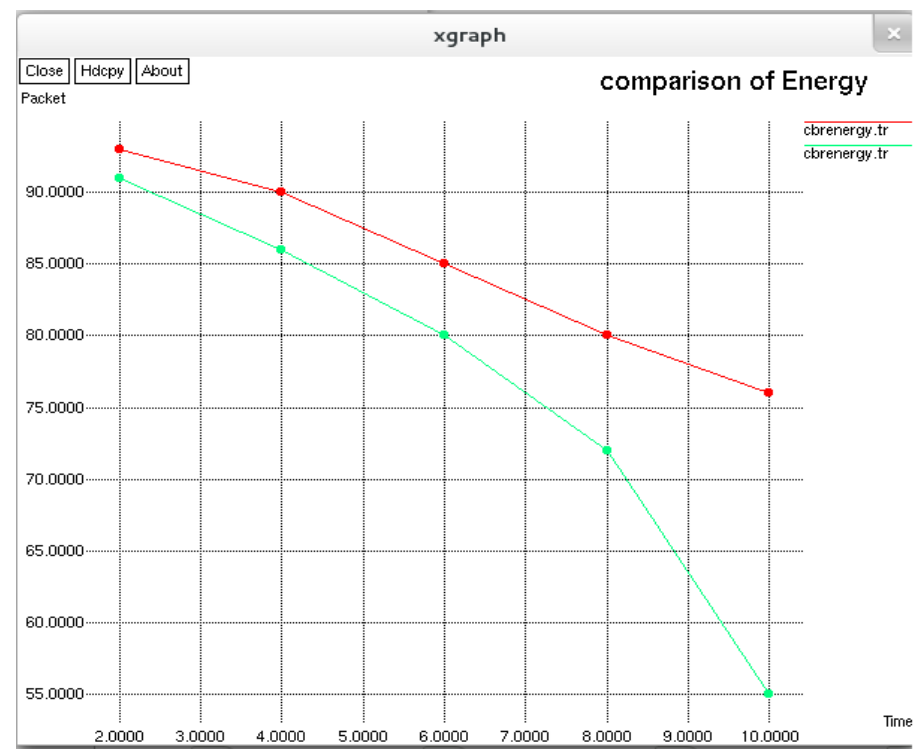

Fig 2 comparison of Energy

3. Delay: The time taken by packet to reach the destination. Total time taken by packet to reach destination is called delay. The total time minus time taken by packet to reach destination is called delay. The formula for : Avg. Delay=S/N where $\mathrm{S}$ is sum of time taken by node to reach the data and $\mathrm{N}$ is total packet received by destination the fig 3 shows that the proposed technique delay is less as compared to previous technique. 
Vol. 7, Issue 9, September 2018

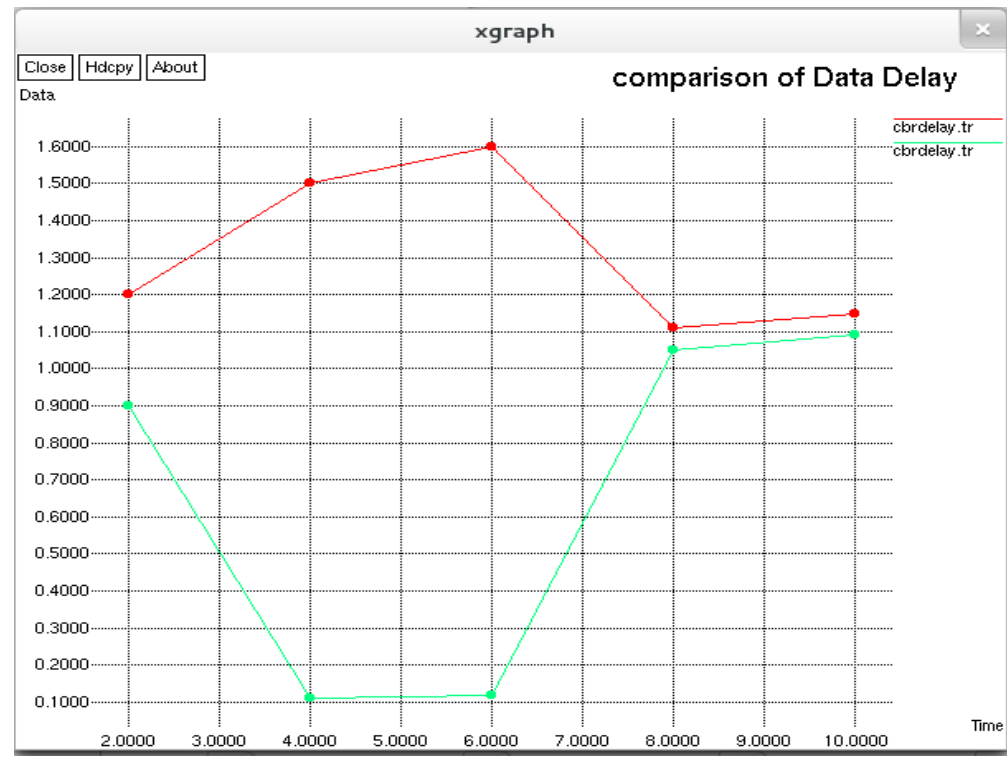

Fig 3 comparison of Delay

\section{CONCLUSION}

This proposed technique shows that performance on the basis of packet delivery factor, delay and energy. By comparing this technique on the basis of various types of performance metrics, factors and parameters then we reach to a conclusion that our proposed technique Conceded Cluster Head Routing Protocol is better than previous technique that is Cluster Based Routing Protocol. To perform better in large scale environment the clustering is proposed. In clustering the mobile node is grouped within range and one mobile node is elected as cluster head which is responsible for transmission in cluster. Future scope is to maintain the overlapping of malicious node so that network has long life.

\section{REFERENCES}

[1]. Iftikhar Hussain, Chen Binagcai "Cluster Formation and Cluster Head Selection Approach for Vehicle Ad-Hoc Network (VANET) using KMeans and Floyd-Warshall Technique" International Journal of Advanced Computer Science and Applications (IJACSA) Vol. 8, Issue 12, 2017.

[2]. Piyalikar, Pritam Kar, Mrinal Kanti Deb Barma "Forecast Weighted Clustering in VANET" Twelfth International Multi-Conference on Information Processing-2016 (IMCIP-2016).

[3]. Vijayanand Kumar, Rajesh Kumar Yadav "Prolonging Network Lifetime by Electing Suitable Cluster Head by Dynamic Weight Adjustment for Weighted Clustering Algorithm in VANET" International Conference on Computing for Sustainable Global Development (2016- INDIACom).

[4]. Renu Popli, kanwal garg, sahil batra "SECHAM: Secure and Efficient Cluster Head Selection Algorithm for VANET", 978-9-3805-44212/16(2016- IEEE).

[5]. K.Gomathi, B.Parvathavarthini “An Efficient Cluster based Key Management Scheme for VANET with Authentication" 978-1-4244-90080/10/2010 IEEE, 17 February 2011.

[6]. Poonam Thakur, Dr. Anita Ganpati “Cluster Based Route Discovery Technique for Routing Protocol in VANET" International Conference on Green Computing and Internet of Things (ICGCloT), October 2015.

[7]. M.Sc.Ali Abdulrahman Mahmood et,al "Modified AODV Routing Protocol to Detect the Black Hole Attack in VANET" International Journal of Advanced Research in Computer Science and Software Engineering Vol.5, Issue 7, July 2015.

[8]. Aditya Upadhyay, Manoj Sindhwani “ Cluster Head Selection Procedure using Priority based Technique in VANET" Indian Journal of Science and Technology Vol. 9, Issue 37, October 2016.

[9]. Tadiparthi Priyanka, T.P. Sharma "A Survey on Clustering Techniques used in Vehicular Ad hoc Network" International Journal of Advanced Computational Engineering and Networking Vol. 2, Issue 8, August 2014.

[10]. Nitika Phull, Dr. Tanupreet Singh Preet "A Review Paper on VANETs" International Journal of Latest Engineering Reseach and Applications (IJLERA) Vol. 2, Issue 4, April 2017.

[11]. Shivani Rana, Swati Rana, Kamlesh C. Purohit et,al "A Review of Various Routing Protocols in Vehicular Ad-Hoc Networks (VANET)" International Journal of Computer Applications, Vol. 96, Issue 18, June2014.

[12]. Chamkaur Singh, Vikas Gupta , Gurmeet Kaur “A Review Paper on Introduction to VANET” International Journal of Engineering Trends and Technology (IJETT) - Vol. 11, Issue 1, May 2014.

[13]. Harpinder Kaur, Navjot kaur, Sandeep Waraich " Comparative Analysis of Clustering Protocols for Wireless Sensor Networks" International Journal of Computer Applications (0975 - 8887) Vol. 115 Issue 1, April 2015.

[14]. D.Satyajeet, A.R.Deshmukh, S.S.Dorle "Hetrogeneous Approaches for Cluster based Routing Protocol in VANET" International Journal of Computer Applications, Vol. 134, Issue 12, January 2016.

[15]. NehaSingla, Ruby Gupta "A Review of Performance Evaluation of the Routing Protocols in VANETs" Vol. 2, Issue 11, November 2014.

[16]. Vaibhav V. Bhujade, Deepak Chaudhary, Suraj V. Raut “ A Review Paper on Behavior of Node in VANET” International Journal on Recent and Innovation Trends in Computing and Communication, Vol. 3, Issue 4, April 2015. 